\title{
Optimal transport- and kernel-based early detection of mild cognitive impairment patients based on magnetic resonance and positron emission tomography images
}

\author{
Ziyu Liu', Travis S. Johnson², Wei Shao ${ }^{2}$, Min Zhang ${ }^{1}$, Jie Zhang ${ }^{3 *}$ and Kun Huang ${ }^{2,4^{*}}$ (D)
}

\begin{abstract}
Background: To help clinicians provide timely treatment and delay disease progression, it is crucial to identify dementia patients during the mild cognitive impairment $(\mathrm{MCl})$ stage and stratify these $\mathrm{MCl}$ patients into early and late $\mathrm{MCl}$ stages before they progress to Alzheimer's disease (AD). In the process of diagnosing $\mathrm{MCl}$ and $\mathrm{AD}$ in living patients, brain scans are collected using neuroimaging technologies such as computed tomography (CT), magnetic resonance imaging (MRI), or positron emission tomography (PET). These brain scans measure the volume and molecular activity within the brain resulting in a very promising avenue to diagnose patients early in a minimally invasive manner.

Methods: We have developed an optimal transport based transfer learning model to discriminate between early and late $\mathrm{MCl}$. Combing this transfer learning model with bootstrap aggregation strategy, we overcome the overfitting problem and improve model stability and prediction accuracy.

Results: With the transfer learning methods that we have developed, we outperform the current state of the art $\mathrm{MCl}$ stage classification frameworks and show that it is crucial to leverage Alzheimer's disease and normal control subjects to accurately predict early and late stage cognitive impairment.
\end{abstract}

Conclusions: Our method is the current state of the art based on benchmark comparisons. This method is a necessary technological stepping stone to widespread clinical usage of MRI-based early detection of AD.

Keywords: Transfer learning, Optimal transport, Bootstrap aggregation

\section{Background}

$\mathrm{AD}$ is an irreversible, degenerative brain disorder, affecting over six million Americans and is the sixth leading cause of death in the USA [1]. AD is hallmarked by neuron loss [2], inflammation [3], amyloid plaques [4], and tau deposition [5], which lead to progressive tissue loss in the brain and cognitive decline in the patient [6].

\section{*Correspondence: jizhan@iu.edu; kunhuang@iu.edu}

${ }^{2}$ Department of Biostatistics and Health Data Science, Indiana University School of Medicine, Indianapolis, USA

${ }^{3}$ Department of Medical and Molecular Genetics, Indiana University School of Medicine, Indianapolis, USA

Full list of author information is available at the end of the article
Diagnosing AD is largely based on tests of cognitive impairment combined with technologies such as CT, MRI, or PET but can only be verified after death on the postmortem brain [7]. Patients who have not yet progressed to $\mathrm{AD}$ may be diagnosed with mild cognitive impairment (MCI). The direct definition of MCI has undergone recent changes. Due to these changes, the diagnostic quality of the MCI designation is only becoming more well refined with improved research into patient stratification [8] and diagnostic guidelines [9]. Despite the fact that $\mathrm{MCI}$ is not a prodromal stage of $\mathrm{AD}$, it is a transitional phase between normal cognitive aging and $\mathrm{AD}$ in which individuals demonstrate objective cognitive impairment

(c) The Author(s). 2022 Open Access This article is licensed under a Creative Commons Attribution 4.0 International License, which permits use, sharing, adaptation, distribution and reproduction in any medium or format, as long as you give appropriate credit to the original author(s) and the source, provide a link to the Creative Commons licence, and indicate if changes were made. The images or other third party material in this article are included in the article's Creative Commons licence, unless indicated otherwise in a credit line to the material. If material is not included in the article's Creative Commons licence and your intended use is not permitted by statutory regulation or exceeds the permitted use, you will need to obtain permission directly from the copyright holder. To view a copy of this licence, visit http://creativecommons.org/licenses/by/4.0/. The Creative Commons Public Domain Dedication waiver (http://creativecommons.org/publicdomain/zero/1.0/) applies to the data made available in this article, unless otherwise stated in a credit line to the data. 
and report subjective complaints but have relatively intact functional abilities [8]. Since early and late stage MCI (E$\mathrm{MCI}$ and L-MCI) have different survival rates and rates of developing $\mathrm{AD}$ [9], to help clinicians to provide timely treatment and delay disease progression, it is crucial to identify E-MCI and L-MCI patients with higher rates of biomarker abnormalities and progression to $\mathrm{AD}$ [8]. In the process of diagnosing $\mathrm{MCI}$ and $\mathrm{AD}$ in living patients, brain scans are collected using neuroimaging technologies such as CT, MRI, and PET to rule out other potential causes of the disease. These brain scans measure the volume and molecular activity within the brain resulting in a very promising avenue to diagnose patients.

Specifically, neuroimaging techniques enable us to identify regions of interests related to $\mathrm{AD}[10]$ and extract sensitive markers for $\mathrm{AD}$. It has been demonstrated that features extracted from structural MRI and PET can help us investigate the neurophysiological feature of $\mathrm{AD}$ and MCI $[11,12]$. These features can be utilized to diagnose the early stage of $\mathrm{AD}$ patients and predict whether an MCI patient will progress to AD [13]. We seek to utilize these features for distinguishing $\mathrm{E}-\mathrm{MCI}$ versus $\mathrm{L}-\mathrm{MCI}$ and formulate this problem as a classification task.

Recent progress in machine learning (ML) and pattern recognition methods shed light on the diagnosis of $\mathrm{AD}$ patients with the help of neuroimaging features. Despite the wide applications of ML models in biomedical problems, there are two major challenges in classifying MCI stages, namely that the collection of multiple-modality datasets is costly and time consuming, and that the effect size observed between E-MCI and L-MCI is too small compared with the feature dimension. This may lead to the overfitting issue, which occurs when the model performs well on training samples while lacks generalizability on unseen data. We seek to enlarge the training sample size to overcome the overfitting problem and improve model stability.

Accordingly, it is of great interest to develop ML models that utilize samples from easier-to-train tasks that are related and have more readily available data. In dementia, the $\mathrm{AD}$ patient versus $\mathrm{NC}$ patient task can be leveraged to transfer knowledge to the more challenging task of predicting MCI stage. Some previous works $[13,14]$ introduced auxiliary tasks such as the AD and NC classification task to identify disease related features and construct the decision function for classification. Transferring knowledge from different but related auxiliary tasks to increase the prediction accuracy on a more difficult target task is a widely used ML strategy called transfer learning (TL). TL uses heterogeneous data and has to face the challenging $\mathrm{ML}$ dilemma as the decision function learned from the source (auxiliary) task cannot be directly applied to the target domain. Two heterogeneous datasets will occupy different distributions in the feature spaces, which is termed distributional drift. Traditional TL techniques adopt sample weighting strategies and feature alignment strategies [15] to overcome the distributional drifting problem. Recently, Optimal Transport (OT) theory has been successfully introduced in TL problems [16, 17]. Since OT has shown great promise in tackling the data drifting (target shifting) issue, we adopt it in our model to address the difficulty of utilizing $\mathrm{AD}$ and $\mathrm{NC}$ samples for tackling our L-MCI and E-MCI stratification problem.

Our model consists of three main components: feature selection, TL, and bootstrap aggregation. We will first use the robust multi-label transfer feature learning rMLTFL [13] framework, which can be used for feature selection as well as the traditional one-way ANOVA to select representative features from MRI and PET data modalities. Then, we will develop the OT TL strategies to train classifiers for stratifying L-MCI and E-MCI with the help of $\mathrm{AD}$ and NC samples. Finally, we will apply the Bootstrap Aggregation (BAg) strategy to overcome the overfitting problem and improve stability and accuracy.

\section{Methods}

\section{Data collection and preprocessing}

The Alzheimer's Disease Neuroimaging Initiative (ADNI) provides researchers with multi-modal longitudinal data for subjects as they work to define the progression of $\mathrm{AD}$. The ADNI-1 dataset contains 202 subjects with MRI and PET brain images. The updated dataset ADNI-2 assessed participants from the ADNI-1 phase besides new participant groups including elderly controls and subjects with significant memory concern, E-MCI, and L-MCI. We summarize the samples used in our study in Table 1.

The feature extraction process includes image registration, region of interests selection, and feature quantification. We specifically use the morphometry features extracted from voxel-based measures of structural MRI (VBM-MRI) and fluorodeoxyglucose positron emission tomography (FDG-PET) images and denote the two classes of features as VBM and FDG features (Additional

Table 1 The values are expressed as mean \pm standard deviation

\begin{tabular}{lllll}
\hline & NC & E-MCI & L-MCI & AD \\
\hline Number & 211 & 273 & 187 & 160 \\
Gender (M/F) & $190 / 101$ & $153 / 119$ & $108 / 76$ & $95 / 65$ \\
Age & $76.1 \pm 6.5$ & $71.5 \pm 7.1$ & $73.9 \pm 8.4$ & $75.2 \pm 7.9$ \\
Education & $16.4 \pm 2.6$ & $16.1 \pm 2.6$ & $16.4 \pm 2.8$ & $15.9 \pm 2.8$ \\
MMSE & $29.0 \pm 1.2$ & $28.4 \pm 1.5$ & $27.7 \pm 1.7$ & $24.0 \pm 2.6$ \\
CDR & $0.0 \pm 0.1$ & $0.5 \pm 0.1$ & $0.5 \pm 0.1$ & $0.7 \pm 0.3$ \\
\hline
\end{tabular}

AD Alzheimer's disease, $N C$ normal control, E-MCl early mild cognitive impairment, L-MCI late mild cognitive impairment, MMSE Mini-Mental State Examination, and $C D R$ clinical dementia rating 
files 1 and 2). The details of feature extraction can be found in the Materials and workflow section of [18].

\section{Feature selection}

To reasonably utilize informative features from the two data modalities, we used the robust multi-label transfer feature learning (rMLTFL) model [13] to filter out features that are irrelevant to the classification task. In the study by Cheng et al. [13], this model was applied to select features to train a support vector machine (SVM) model for distinguishing Progressive MCI (P-MCI) and Stable MCI (S-MCI). This framework can help identify features related to the target task (L-MCI vs E-MCI) that benefit from auxiliary tasks ( $\mathrm{AD}$ vs $\mathrm{NC}, \mathrm{AD}$ vs $\mathrm{MCI}, \mathrm{MCI}$ vs NC). However, it faces a difficult situation that separating E-MCI and L-MCI samples using linear SVM and logistic regression (LR) is not effective, even with multiple kernels. Therefore, we only adopted it as a feature selection method and compared it with the traditional one-way analysis of variance (ANOVA) feature selection technique.

We denote the dataset on the target task (L-MCI vs E-MCI) as $\left(\mathbf{X}^{1}, \mathbf{X}^{2}, \mathbf{y}^{t}\right) . \mathbf{X}^{1}, \mathbf{X}^{2} \in \mathbb{R}^{460 \times 116}$ represent the FDG and VBM features respectively while $\mathbf{y}^{t} \in\{-1,+1\}$ is the class label correspond to E-MCI and L-MCI respectively. We also construct three auxiliary domains $\left\{\left(\mathbf{A}_{1}^{1}, \mathbf{A}_{1}^{2}, \mathbf{y}_{1}^{a}\right),\left(\mathbf{A}_{2}^{1}, \mathbf{A}_{2}^{2}, \mathbf{y}_{2}^{a}\right),\left(\mathbf{A}_{3}^{1}, \mathbf{A}_{3}^{2}, \mathbf{y}_{3}^{a}\right)\right\}$. Each triplet in the bracket represents a task that may be helpful for feature selection. For instance, $\left(\mathbf{A}_{2}^{1}, \mathbf{A}_{2}^{2}, \mathbf{y}_{2}^{a}\right)$ denotes the FDG and VBM features along with labels for AD $(+1)$ and NC (1) patients. To construct a multi-bit label coding matrix for the TL task, we firstly trained three logistic regression models on three auxiliary domains. Then, we used these three models to independently estimate three labels for each patient on the target domain. Finally, we concatenated the true label with three predicted labels to form a multi-bit label for each patient and obtain a multi-bit label matrix $\mathbf{Y}=\left[\mathbf{y}^{t}, \mathbf{y}_{1}^{p}, \mathbf{y}_{2}^{p}, \mathbf{y}_{3}^{p}\right] \in \mathbb{R}^{460 \times 4}$ (one true label, three predictions). The goal of the rMLTFL algorithms is to learn a weight matrix $\mathbf{W}=\left[\mathbf{w}^{t}, \mathbf{w}_{1}, \mathbf{w}_{2}, \mathbf{w}_{3}\right] \in \mathbb{R}^{116 \times 4}$ which can be decomposed into two components $\mathbf{P}$ and $\mathbf{Q}$ for feature selection and domain identification respectively. Specifically, the objective function is formulated a following:

$$
\begin{array}{r}
\min _{\mathbf{W}, \mathbf{P}, \mathbf{Q}}\|\mathbf{Y}-\mathbf{X} \mathbf{W}\|_{F}^{2}+\lambda_{1}\|\mathbf{P}\|_{2,1}+\lambda_{2}\left\|\mathbf{Q}^{T}\right\|_{2,1}+ \\
\lambda_{3} \sum_{i=1}^{3}\left\|\left(\mathbf{X w}^{t}-\mathbf{X w}_{i}\right)-\left(\mathbf{y}^{t}-\mathbf{y}_{i}^{p}\right)\right\|_{2}^{2}, \\
\text { s.t. } \mathbf{W}=\mathbf{P}+\mathbf{Q}
\end{array}
$$

The first term is to ensure the similarity between the multi-bit labels $\mathbf{Y}$ and its prediction $\mathrm{XW}$. In the second and the third term, we use the 2,1 norm to capture the shared features across all tasks and filter out the unrelated tasks. The 2,1 norm forces some rows of $\mathbf{P}$ and some columns of $\mathbf{Q}$ to be all zero. Non-zeros rows in $\mathbf{P}$ and nonzero columns in $\mathbf{Q}$ corresponds to informative features and tasks respectively. The last term indicates that the distance from predicted target domain label $\mathbf{X w}^{t}$ to multi-bit label $\mathbf{X} \mathbf{w}_{i}^{p}$ should be similar to the distance from the true label $\mathbf{y}^{t}$ to the estimated multi-bit label $\mathbf{y}_{i}^{p}$.

The above rMLTFL framework to select feature can be illustrated in Fig. 1. After we obtained the multi-bit label matrix $\mathbf{Y}$, we used the accelerate gradient descent algorithm to optimize the target function (1). Then, we filtered out domains that corresponded to all zero columns in $\mathbf{Q}$. After that, we repeated the same process as above without these useless domains. Finally, we selected rows that corresponded to non-zero rows in $\mathbf{P}$ as features related to the target task. When implementing rMLTFL and oneway ANOVA to select features, we applied each method to the two data modalities separately and simultaneously. Hence, we obtained six sets of sample features. After examining the prediction performance of these feature sets, we chose the most relevant feature sets and achieved higher prediction accuracy by applying model aggregation techniques.

\section{Optimal transport for transfer learning}

In previous work of MCI stage classification, i.e., classifying P-MCI vs S-MCI [13] and MCI converters versus MCI non-converters [14], a common assumption is that

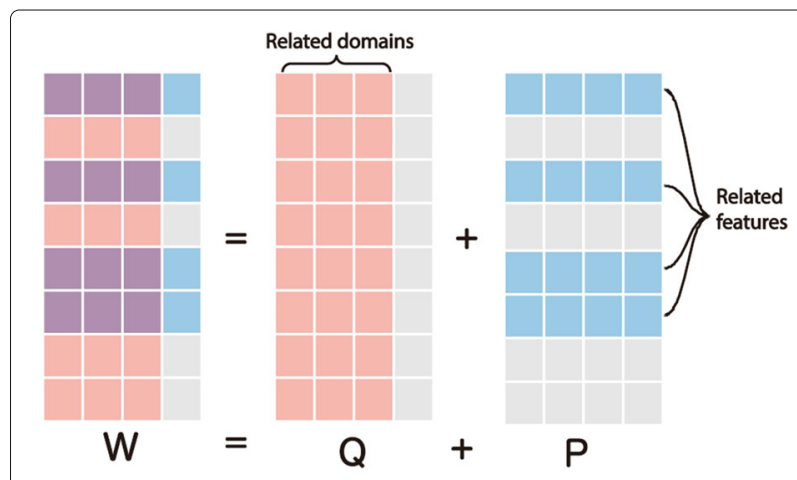

Fig. 1 The learnable weight matrix $\mathbf{W}$ can be decomposed into two matrices, $\mathbf{Q}$ and $\mathbf{P}$. They are responsible for selecting target problem related tasks ( $A D$ vs NC, AD vs $\mathrm{MCl}, \mathrm{MCl}$ vs NC) and features. By enforcing the $12-11$ norm of $\mathbf{Q}^{T}$ and $\mathbf{P}$ to be small, these group lasso penalty terms on rows on $\mathbf{P}$ and columns of $\mathbf{Q}$ encourage the rows of $\mathbf{P}$ and columns of $\mathbf{Q}$ in (1) to have all zero (rows and columns in gray) or non-zero elements. The first column of $\mathbf{Q}$ corresponds to the L-MC vs E-MCl stratification task and the rest of them correspond to three auxiliary tasks. We could observe from the plot that the AD v NC and the $A D$ vs $M C l$ tasks are two related domains while the $M C I$ vs NC task could not provide helpful information. Similarly, non-zero rows of $\mathbf{P}$ capture the shared features among useful domains 
introducing auxiliary tasks (ie. AD vs NC) can improve the accuracy of classification. It is assumed that at least some of these auxiliary domains can help us understand the target domain, even without feature transformation. From the t-distributed stochastic neighbor embedding ( $t$ $\mathrm{SNE}$ ), boxplot of principal components, and violin plot of features we conclude that the feature distribution of L$\mathrm{MCI}$ and $\mathrm{E}-\mathrm{MCI}$ is similar to the pattern of those in the $\mathrm{AD}$ and $\mathrm{NC}$ subjects. However, the difference between early and late stage $\mathrm{MCI}$ is much more subtle than the difference between $\mathrm{AD}$ and $\mathrm{NC}$ samples. Therefore, we must adopt TL strategies to reduce the inter-task discrepancy between $\mathrm{AD}$ vs NC task and E-MCI vs L-MCI task while maximizing the intra-task differences. Traditional TL methods using sample weighting or feature alignment strategies to adapt source data samples (i.e. AD and NC samples) to the target domain (i.e., L-MCI and E-MCI samples)[15]. Compared with these previous works, the OT for TL frameworks can capture the intrinsic geometry structure difference of two feature spaces and address the distributional drift problem more efficiently. We illustrate in our experiments that our proposed method based on OT outperforms the current state-of-the-art methods.

OT maps samples from one domain to another by minimizing the earth mover's distance $[16,19]$ between sample distributions in two domains. To better understand the feature distribution within and across classes and to estimate a better transformation, $[16,17,20]$ added different regularization terms such as $L_{1} l_{2}$ and $L_{p} l_{1}$ terms to achieve group sparsity. By adding the group sparsity regularization terms, the OT feature mapping strategy only projects $\mathrm{L}-\mathrm{MCI}$ training samples to the $\mathrm{AD}$ samples and E-MCI training samples to the NC samples. For computational efficiency, most of the state-of-the-art OT models incorporate an entropy regularization term. This regularized version of earth mover's distance [21] is call Sinkhorn distance (SD). In this study, we implemented three OT mapping strategies defined by SD, SD with $L_{p} l_{1}$ regularization term, and SD with $L_{1} l_{2}$ regularization term respectively.

Before introducing the experiment setting of using OT to train classifiers, we want to emphasize the difference between our proposed method and traditional OT methods for TL that are used as benchmarks in this study. Traditionally, the source domain (AD vs NC) features are mapped to the target domain (L-MCI vs E-MCI) via an OT strategy. Then, AD and NC labels as well as the transformed features can be used to train a classifier on the target domain that will be directly applied to the L-MCI vs E-MCI stratification task. This strategy is powerful when dealing with the condition that few labels are available on the target domain and the decision boundary for the target task is easy to learn. In our problem, the intrinsic difficulty is that the decision boundary is difficult to learn even after using kernel methods. Fortunately, we have plenty of samples (187 L-MCI, 273 E-MCI) on the target domain, which enable us to separate them into training and testing sets. Therefore we instead map training samples on the target domain(L-MCI vs E-MCI) to the source domain (AD vs $\mathrm{NC}$ ) where the classification boundary is more clearly defined. During this process, we learn a non-linear OT mapping strategy $\mathbf{T}$. Then, we train classifiers to use $\mathrm{AD}$ and $\mathrm{NC}$ samples as well as E-MCI and L-MCI samples transformed by $\mathbf{T}$. After that, we use the OT mapping $\mathbf{T}$ to project testing samples to the source domain and use the classifier to stratify E-MCI and L-MCI samples. Finally, we evaluate the classification performance using accuracy and area under the receiver operating curve (AUC) score. Figure 2 illustrates the effects of using OT to obtain more distinguishable features in synthesized data.

In our experiments on real AD data, we investigate different OT mapping strategies as well as different classifiers on the source domain. In Fig. 3, we illustrate how to adapt MCI samples onto the $\mathrm{AD}$ and $\mathrm{NC}$ domain. In Fig. 5 panel (A), we demonstrate how to combine different OT mapping strategies with different classifiers. Since logistic regression achieves higher prediction accuracy than SVM, we adopt it as a benchmark classifier and combine it with linear and polynomial kernel functions to form kernel based classifiers.

\section{Bootstrap aggregation to improve model stability}

Bootstrap aggregation (BAg) is an algorithm proposed in [22] for both regression and statistical classification. By randomly sampling training sets (bootstrapping) with replacement, one can train several classifiers using the same algorithm. By aggregating model predictions based on the majority voting strategy or aggregating prediction probabilities, we raise the stability of our models by reducing inter-model variability from overfitting. When we implemented the BAg strategy, we first need to decide the number of "bags" to use. Since our study only contained a few hundreds samples, We used 5 bags to train five submodels. Then, we aggregated the model using a majority vote strategy. The prediction probability was obtained by calculating the mean prediction probability across each sub-model. We illustrated the pipeline in Fig. 3.

\section{Feature selection comparison}

Using one-way ANOVA, we calculated the $p$ value for each feature individually. Using the $p$ value threshold 0.05 , we selected 47 out of 116 features from the FDG and the VBM data modality respectively. The rMLTFL method captures features by training a model and selecting features based on that trained model. We need to verify the stability of this feature selection procedure. To determine which hyper-parameters to use and whether the collection of useful features were dependent on the training set, 

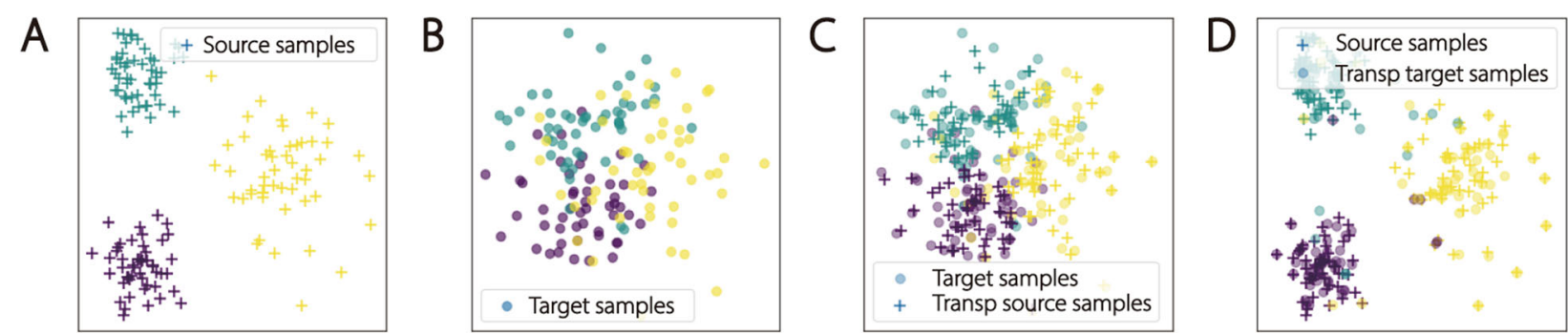

Fig. 2 We use a synthetic Gaussian distributed dataset to demonstrate our method. In panel $\mathbf{A}$, we generate three clusters of gaussian distributed samples. Their clusters are distinct, hence simple decision boundaries can separate them clearly. This example corresponds to the AD vs NC classification task. In panel $\mathbf{B}$, we also generate three clusters which are not distinctive from one another. In fact, the E-MCl and L-MCl clusters are much less distinct than the samples in panel $\mathbf{B}$. In panel $\mathbf{C}$, we use OT to map the source domain samples onto the target domain. In the last panel (D), we use our proposed method adopting OT to map target samples onto the source domain by utilizing sample labels

we used five-fold cross-validation to verify the robustness of the rMLTFL method. We took a grid search approach for the three hyper-parameters over 1000 combinations of these parameters and chose the hyper-parameter combination with the highest average prediction accuracy. Using the optimal hyper-parameters, we ran the rMLTFL algorithm on the FDG data modality to filter out useless features and obtained 96 features by merging selected features respectively across five folds. For the VBM data modality, the model only filtered out one useless features over all hyper-parameter combinations. Therefore, we kept 115 feature from the VBM data modality. To combine the two data modalities, we concatenated the two feature vectors and repeated the same process as described above. We visualized the selected features ability to stratify $\mathrm{NC}$, $\mathrm{E}-\mathrm{MCI}, \mathrm{L}-\mathrm{MCI}$, and $\mathrm{AD}$ individually and aggregated via PCA and tSNE plots.

\section{Transfer learning benchmark comparison}

First we benchmarked different OT mapping strategies via ten-fold cross-validation on each data modality individually and the combined data modalities. Specifically, we applied three different OT mapping regularization strategies: SD (OT regularized by an entropy regularization term), SD regularized by $L_{p} l_{1}$ norm, and SD regularized by $L_{1} l_{2}$ norm to map samples from the target domain to the source domain. The usage of these regularization norms is to enforce intra-class similarity and improve computational efficiency. When we mapped L$\mathrm{MCI}$ and $\mathrm{E}-\mathrm{MCI}$ samples to the domain of $\mathrm{AD}$ and $\mathrm{NC}$ samples, we utilized the labels of training samples, i.e. E-MCI and L-MCI. Using these transformed samples as well as $\mathrm{AD}$ and $\mathrm{NC}$ samples, we evaluated the performance on the source domain via accuracy and AUC scores.

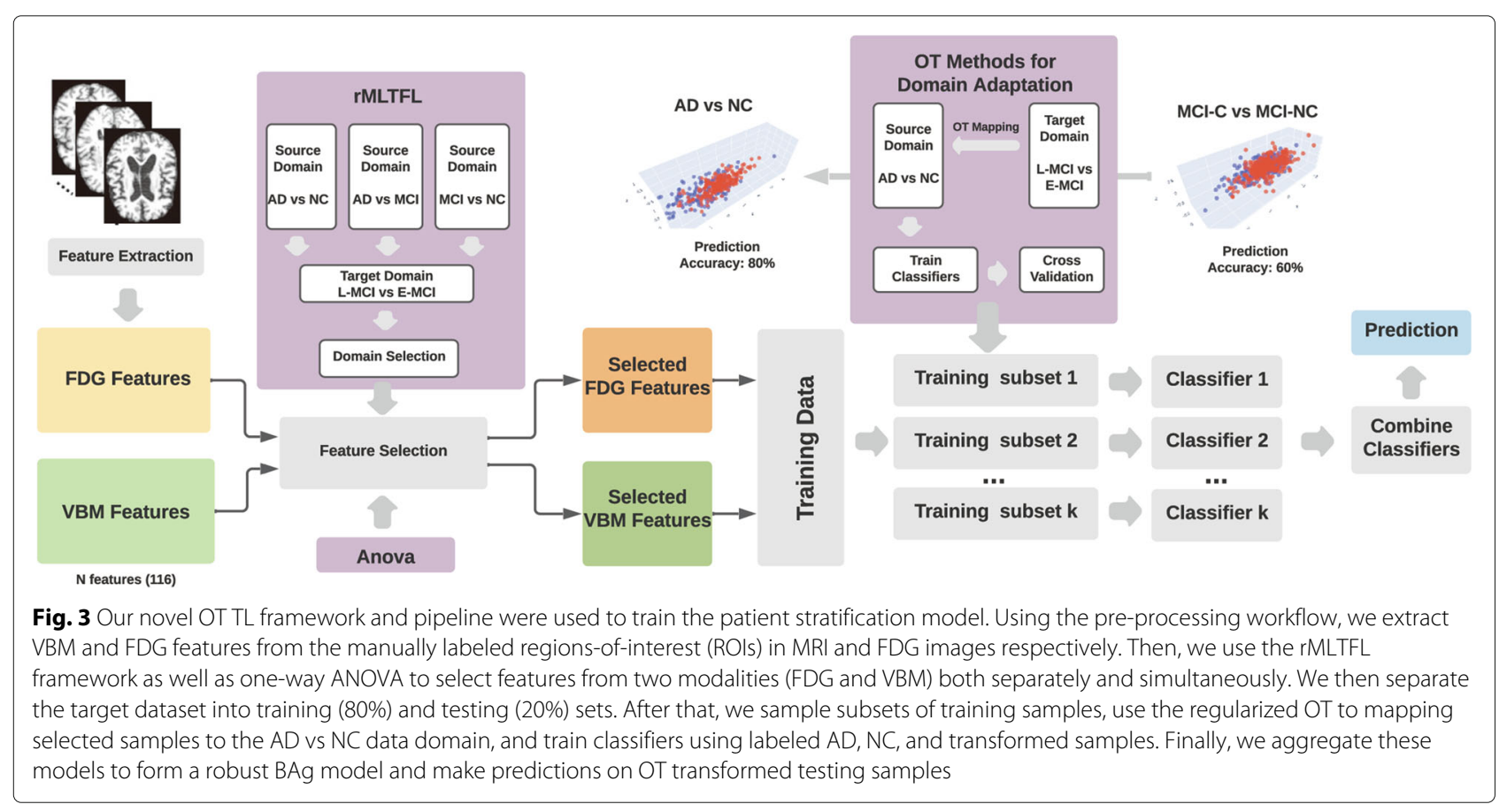


Besides two baseline methods and the rMLTFL framework, we also compared our model with other TL benchmarks and multiple kernel learning strategies. For TL benchmarks, we compared our method with: Importanceweighting with logistic discrimination (IW) [23], Transfer Component Analysis (TCA) [24], Semi-supervised Subspace Alignment (SUBA) [25], Feature-Level Domain Adaptation (FLDA) [26], and Boosting for Transfer learning (TrAdaBoost)[27]. We also compared with multiple kernel learning strategies including: the simple average of base kernels (AverageMKL), margin-based combination of kernels (EasyMKL) [28], radius-margin ratio optimization for dot-product boolean kernel learning (GRAM) [29], margin and radius based multiple kernel learning (RMKL) [30], simple but effective methods for combining kernels in computational biology (PWMK) [31], and centered kernel alignment optimization in closed form (CKA) [32]. Since we use the decision tree as a basic classifier for some of these benchmarks, e.g. TrAdaBoost, we can't obtain the AUC score directly. To evaluate the model performance, we use ten-fold cross-validation and calculate the average and standard deviation of the accuracy score.

\section{Bootstrap aggregation comparison}

We separated the dataset into training and testing sets (80\% and $20 \%)$. On the training set, we implemented the bootstrap strategy in a slightly different manner. During the stage of bootstrapping, we randomly split the training set into five folds and picked four folds each time to train a classifier using our OT TL strategy. To demonstrate that our OT alignment improves the stratification performance, we also compared our method with different versions of BAg using traditional SVM, logistic regression, and rMLTFL models as classifiers.

\section{Results}

\section{Diagnostic value of MRI features}

We visualized the selected VBM features in Fig. 4. Panels $\mathbf{A}$ and $\mathbf{B}$ show the t-SNE plots of features selected by ANOVA and rMLTFL respectively. In panel $\mathbf{A}$, we observed that $\mathrm{AD}$ patients mainly concentrated on the upper right corner where L-MCI patient is also denser than other areas while E-MCL and NC samples are denser at the lower left corner. We concluded that the pattern of $\mathrm{AD}$ vs NC may help us delineate the distributions of L$\mathrm{MCI}$ versus E-MCI. The same pattern can be observed in panel $\mathbf{B}$. Panel $\mathbf{C}$ and $\mathbf{E}$ illustrate distributions of first two principle components of ANOVA and rMLTFL features. From these plots we concluded that the distributional differences between the first principle components of L-MCI and E-MCI patients are more subtle than the differences between $\mathrm{AD}$ and $\mathrm{NC}$ patients. $\mathrm{AD}$ and L-MCI patients tended to have lower PC 1 while E-MCI and NC tend to have higher values of PC 1 . We also visualized part of features selected by ANOVA and RMLTFL in D and $\mathbf{F}$. From them we observed the same pattern as the boxplots.

\section{Transfer learning benchmark comparison results}

The results of cross-validation for FDG and VBM data modalities in Tables 2 and 3 demonstrate that our framework outperformed all baseline methods and the original rMLTFL model by increasing prediction accuracy and reducing variability. Based on FDG features, our model achieved $68.76 \pm 7.53 \%$ accuracy and $0.66 \pm$ 0.08 AUC score across ten-fold cross-validation. The SVM and logistic regression baseline methods achieved $61.20 \pm 7.22 \%$ and $64.40 \pm 7.60 \%$ accuracy respectively. Our model also outperformed them on the VBM data modality. Comparing the performance of features selected by rMLTFL and ANOVA, we observed that the rMLTFL features are always superior than ANOVA features. This indicates that even features that are not significant statistically may be helpful to model complex nonlinear differences between sample classes. Combining two data modalities by directly concatenating features did not help us in distinguishing L-MCI and E-MCI patients.

Based on the TL benchmark experiments, our method proved superior to all of these benchmarks (Table 3, Fig. 5). One notable fact is that most of them did not even beat the baseline method logistic regression with linear kernel function. Therefore, traditional TL techniques such as sample weighting and feature alignment strategies may not be effective for us to delineate the distribution patterns of L-MCI and E-MCI. Since our method compared distributions directly, we could glean more information from $\mathrm{AD}$ and NC patients as well as MCI patients in the training set. We also found that Easy MKL, average KL, and PWMK methods yielded relatively high performance on both domains. We concluded that combining multiple kernel functions in an appropriate manner can improve the classification performance.

\section{Bootstrap aggregation comparison results}

In Table 4, we list the aggregated model performance of the testing set for different models and different data modalities. Besides our OT mapping strategies, we also implemented the BAg using two baseline methods and the rMLTFL benchmark method. The performance of our model was significantly superior than SVM, logistic regression, and rMLTFL (Figs. 6 and 7). By choosing different training sets, our model captured heterogeneous patterns. When we aggregated them using a voting strategy, most models could correctly prediction the testing samples. Hence, the accuracy as well as AUC score was much higher than the single model case. On the other hand, the logistic regression, SVM, and rMLTFL 
A

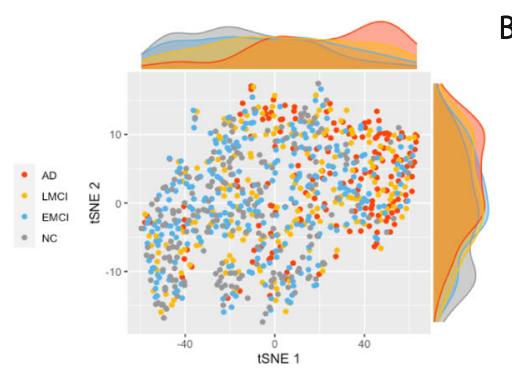



C

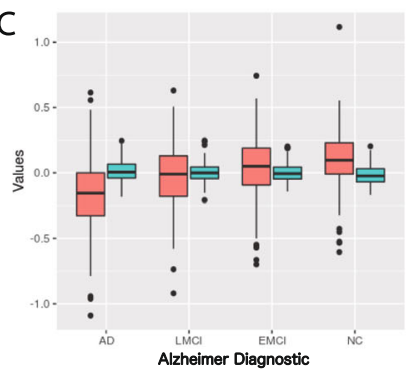

E

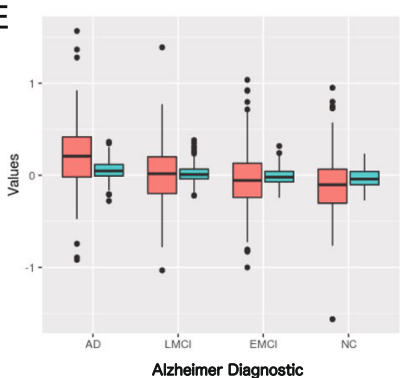

D

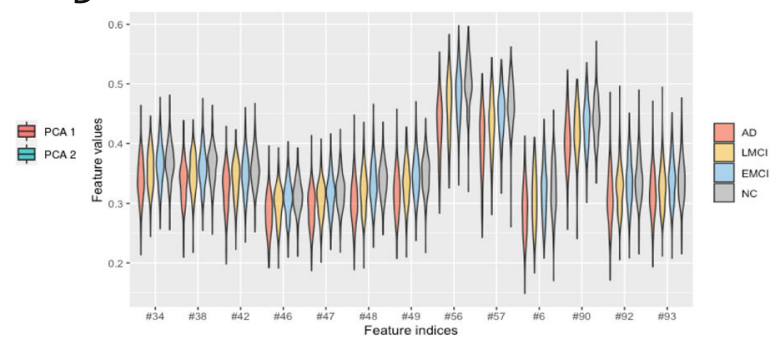

$\mathrm{F}$

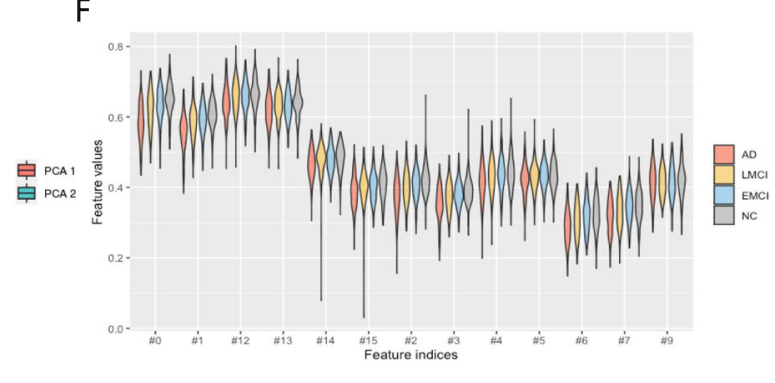

Fig. $4 \mathbf{A}$ and $\mathbf{B}$ represent t-SNE plots and their marginal distributions for VBM features selected by ANOVA and rMLTFL respectively. C and E are box-plots for first two principle components of these selected features. We also visualize the distribution of feature contributions (input feature values after preprocessing to [0,1] scale) selected by ANOVA (D) and rMLTFL (F)

models were quite stable with regard to the training set (Figs. 6 and 7). The patterns they learned are quite homogeneous. We conclude that learning sub-models does not improve model performance for these baseline and benchmark methods.

In order to evaluate the reproducibility of our proposed method, we further split the dataset into three subsets, namely training (80\%), validation (10\%), and testing (10\%) datasets. By training and aggregating models based on training samples and testing on the validation and testing datasets, we obtain AUC curves in Fig. 8. It implies that our model can yield plausible and stable results (highest AUC score $=0.77$ on the validation dataset and 0.78 on the testing dataset) regardless how do we split the dataset.

Table 2 Accuracy (ACC) and AUC score of models based on features selected by rMLTFL and ANOVA ( $p$ value threshold=0.05) respectively

\begin{tabular}{|c|c|c|c|c|c|c|c|}
\hline & & \multicolumn{2}{|c|}{ Sinkhorn distance } & \multicolumn{2}{|c|}{ Sinkhorn distance $+L_{1} I_{2}$} & \multicolumn{2}{|c|}{ Sinkhorn distance $+L_{p} I_{1}$} \\
\hline & & $\overline{A C C}$ & AUC & $\overline{A C C}$ & AUC & ACC & AUC \\
\hline \multirow[t]{2}{*}{ FDG } & rMLTFL & $68.76 \pm 7.53$ & $0.66 \pm 0.08$ & $66.04 \pm 7.53$ & $0.65 \pm 0.08$ & $65.48 \pm 5.04$ & $0.64 \pm 0.07$ \\
\hline & ANOVA & $66.07 \pm 6.96$ & $0.65 \pm 0.07$ & $63.63 \pm 6.01$ & $0.64 \pm 0.07$ & $59.50 \pm 6.53$ & $0.62 \pm 0.08$ \\
\hline \multirow[t]{2}{*}{ VBM } & rMLTFL & $62.37 \pm 6.88$ & $0.62 \pm 0.11$ & $62.74 \pm 0.08$ & $0.62 \pm 0.11$ & $57.86 \pm 6.32$ & $0.60 \pm 0.07$ \\
\hline & ANOVA & $58.94 \pm 7.82$ & $0.59 \pm 0.12$ & $58.68 \pm 0.08$ & $0.58 \pm 0.12$ & $56.79 \pm 0.11$ & $0.58 \pm 0.13$ \\
\hline \multirow[t]{2}{*}{$F D G+V B M$} & rMLTFL & $62.26 \pm 6.48$ & $0.63 \pm 0.05$ & $66.61 \pm 6.29$ & $0.65 \pm 0.06$ & $66.05 \pm 5.91$ & $0.65 \pm 0.08$ \\
\hline & ANOVA & $61.44 \pm 6.23$ & $0.64 \pm 0.05$ & $63.87 \pm 5.75$ & $0.63 \pm 0.06$ & $61.15 \pm 8.01$ & $0.64 \pm 0.07$ \\
\hline
\end{tabular}

The values are denoted as mean \pm standard deviation. We investigated different OT mapping strategies, e.g., using Sinkhorn distance, Sinkhorn Distance with $L_{p} l_{1}$ regularization term, and Sinkhorn distance with $L_{1} l_{2}$ regularization term. Accuracy and AUC scores are calculated by averaging over performances of ten-fold cross-validation on the training set. We exam the model performance on FDG and VBM features separately and simultaneously. 
Table 3 Accuracy of baseline, transfer learning and Multi-kernel benchmark methods

\begin{tabular}{lll}
\hline Methods & FDG & VBM \\
\hline SVM & $61.20 \pm 7.22$ & $57.64 \pm 5.89$ \\
Logistic Reg & $64.40 \pm 7.60$ & $58.72 \pm 6.98$ \\
rMLTFL & $63.33 \pm 9.02$ & $62.53 \pm 9.08$ \\
IW & $60.10 \pm 8.41$ & $59.56 \pm 7.49$ \\
TCA & $59.83 \pm 6.02$ & $57.02 \pm 8.27$ \\
SUBA & $64.68 \pm 4.34$ & $52.44 \pm 8.33$ \\
RBA & $61.46 \pm 8.21$ & $58.17 \pm 8.02$ \\
FLDA & $63.90 \pm 10.00$ & $60.11 \pm 9.05$ \\
TrAdaBoost & $61.45 \pm 8.56$ & $58.98 \pm 7.43$ \\
Easy MKL & $64.72 \pm 9.75$ & $60.38 \pm 7.46$ \\
Average MKL & $63.34 \pm 9.08$ & $60.11 \pm 7.14$ \\
PWMK & $64.19 \pm 9.80$ & $60.11 \pm 7.14$ \\
GRAM & $64.72 \pm 9.75$ & $/$ \\
RMKL & $63.91 \pm 9.53$ & $60.11 \pm 7.14$ \\
CKA & $59.56 \pm 7.49$ & $59.56 \pm 7.49$ \\
\hline
\end{tabular}

The values are denoted as mean \pm standard deviation
Due to the limitation of ADNI patient labels, we cannot perform experiments on other interesting tasks such as P$\mathrm{MCI}$ versus S-MCI. But we added two more experiments on $\mathrm{AD}$ vs $\mathrm{MCI}$ and MCI vs NC to illustrate the effectiveness of our framework. We demonstrate our results and benchmark studies in the supplementary material (Table S1 and S2).

\section{Discussion}

We present our novel method which uses optimal transport to improve the performance discriminating between (E-MCI vs L-MCI) using MRI and PET images. We found that by using OT theory to project the more difficult task, $\mathrm{E}-\mathrm{MCI}$ vs L-MCI, onto the easier task of distinguishing $\mathrm{AD}$ and $\mathrm{NC}$, we were able to achieve higher performance than by using MCI samples alone. This represents not only a significant advance in OT and TL methods but also has clear clinical implications.

Indeed, identifying cognitively impaired individuals early will likely their health outcomes because of early access to treatment and monitoring [33, 34]. These early detection systems are most frequently focused

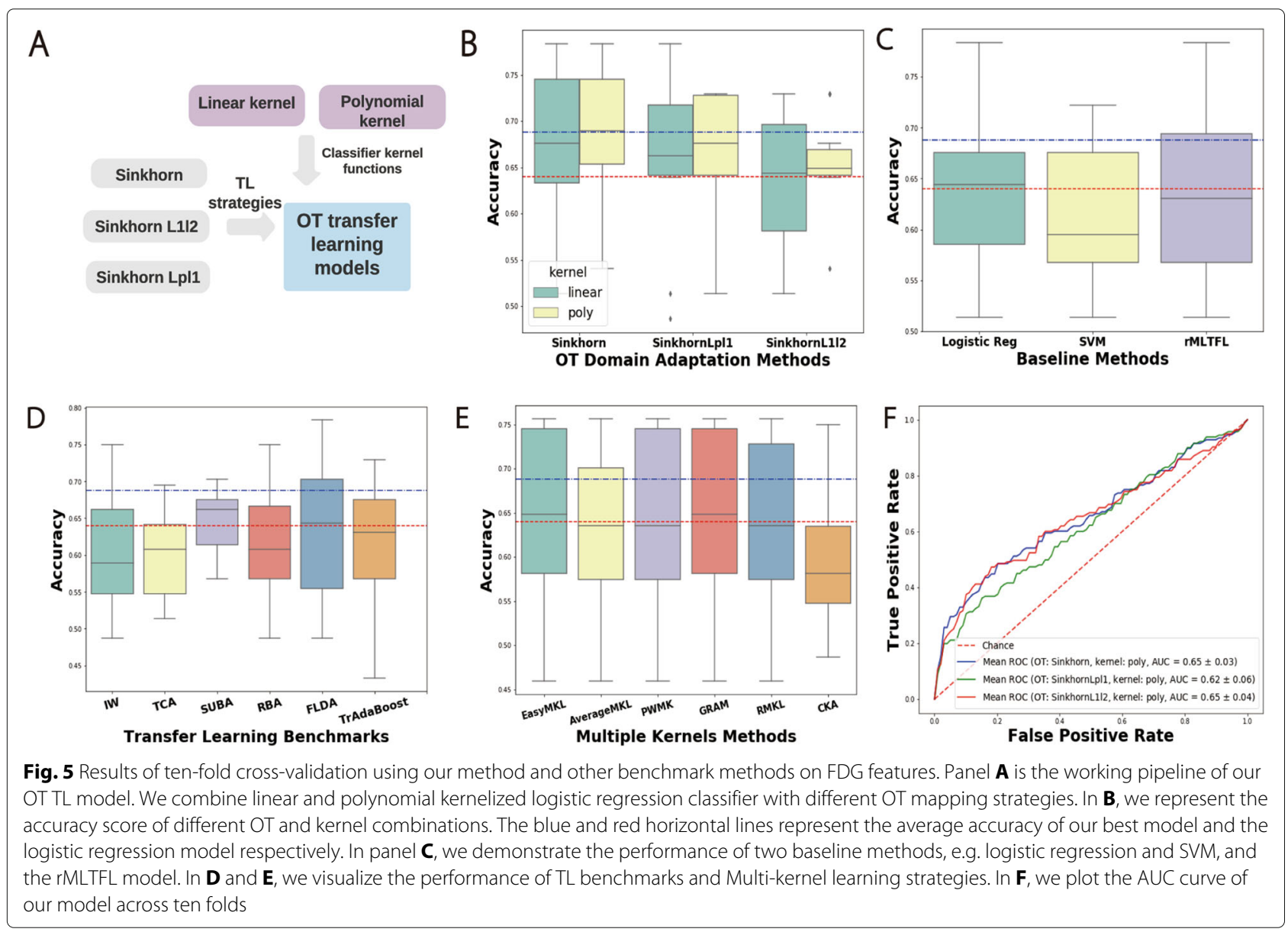


Table 4 Accuracy (ACC) and AUC score of BAg results

\begin{tabular}{|c|c|c|c|c|c|c|}
\hline \multirow[t]{2}{*}{ Methods } & \multicolumn{2}{|l|}{ FDG } & \multicolumn{2}{|l|}{ VBM } & \multicolumn{2}{|c|}{$\mathrm{FDG}+\mathrm{VBM}$} \\
\hline & ACC & AUC & ACC & AUC & ACC & AUC \\
\hline $\mathrm{SD} / \mathrm{I}$ & 72.82 & 0.79 & 68.48 & 0.67 & 70.65 & 0.74 \\
\hline $\mathrm{SD} / \mathrm{p}$ & 73.91 & 0.76 & 64.13 & 0.67 & 69.56 & 0.71 \\
\hline $\mathrm{SD} L_{1} / 2 / \mathrm{l}$ & 75.00 & 0.77 & 67.39 & 0.67 & 69.56 & 0.77 \\
\hline $\mathrm{SD} L_{1} I_{2} / \mathrm{p}$ & 71.74 & 0.76 & 63.04 & 0.67 & 65.21 & 0.70 \\
\hline SD $L_{p} l_{1} / I$ & 71.74 & 0.76 & 59.78 & 0.68 & 73.91 & 0.76 \\
\hline $\mathrm{SD} L_{p} l_{1} / p$ & 71.74 & 0.74 & 67.39 & 0.69 & 66.30 & 0.71 \\
\hline SVM & 57.61 & 0.47 & 57.61 & 0.29 & 57.61 & 0.55 \\
\hline logistic & 68.47 & 0.67 & 58.70 & 0.70 & 67.39 & 0.66 \\
\hline rMLTFL & 63.04 & 0.69 & 60.87 & 0.70 & 63.04 & 0.67 \\
\hline
\end{tabular}

The OT method and kernel function combination is denoted as OT method/kernel function. I and p represent linear and polynomial kernel respectively

on the readily available and minimally invasive medical imaging procedures like MRI and PET scans. Ideally, at risk patients could regularly be tested for $\mathrm{AD}$ and $\mathrm{MCI}$ by their physicians. These imaging technologies offer a potential avenue to a minimally invasive test for cognitive impairment. These clinical tests however are dependent on accurate ML models which can effectively discriminate between cognitively normal, end stage Alzheimers, and the entire spectrum in between.

By using OT to map E-MCI and L-MCI samples to the auxiliary domain, we reduce the inter-task discrepancy between $\mathrm{AD}$ vs NC task and E-MCI vs L-MCI task while maximizing the intra-task differences. This TL technique enable us to train LR classifiers which can stratify E-MCI and L-MCI patients more accurately. We then aggregate sub-TL models using a majority voting strategy to improve the model stability and avoid the overfitting issue.

With the novel methods that we have developed, we outperform the current state-of-the-art TL methods and show that it is crucial to leverage $\mathrm{AD}$ and $\mathrm{NC}$ data to accurately predict L-MCI and E-MCI patients. Such continued improvements are necessary to improve the personal, healthcare, and economic costs [35] associated with over six million AD patients in the USA alone.

\section{Limitations}

When compared with other benchmark works, our model yields a high prediction accuracy and AUC score. We also acknowledge several limitations. Our feature selection method rMLTFL depends on three hyper-parameters. It's of crucial importance to select correct combination hyper-parameters. Although we grid search them over 1000 combinations, there is still lack of evidence that the selected combination is an optimal choice. Furthermore, we have not considered its performance in other challenging $\mathrm{MCI}$ classification tasks such as the $\mathrm{P}-\mathrm{MCI}$ and S-MCI classification task [13]. Finally, our framework is developed based on the VBM and FDG features extracted in [18], which have been exploited in some related studies. Currently the reported performances of all these studies are not good enough for clinical treatment. Potential strategies for improving stratification performance include (1) using more samples for training when more samples are available in the ADNI dataset; (2) since VBM and FDG features extracted by [18] may not be representative enough for distinguishing different $\mathrm{MCI}$ conditions, we could try to adopt more advance feature extracting pipelines; and (3) combining image features with genotype profiles for more accurate assessment. Since genotype data may provide supplementary information to image data, we could train more accurate and stable models based on combining these two heterogeneous data modalities.

\section{Conclusion}

We have developed an optimal transport based transfer learning model to discriminate between E-MCI and L-MCI patients. Our methods are both novel and the current state of the art based on benchmark comparisons. This method is a necessary technological stepping stone to widespread clinical usage of MRI based early detection of $\mathrm{AD}$.
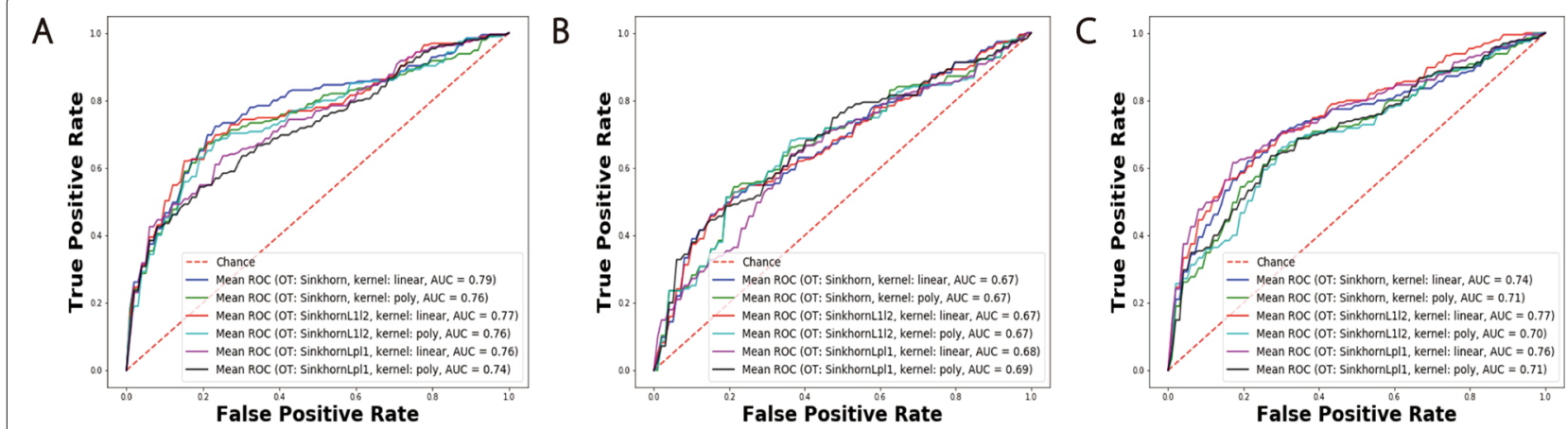

Fig. 6 AUC curves of BAg of our OT transfer learning framework. Panels A, B, and $\mathbf{C}$ correspond to results on the FDG, VBM, and combination of two data modalities respectively. The highest AUC curve is achieved by using SD combined with $L_{1} / 2$ regularization term as OT mapping cost function and linear kernel logistic regression as classifier 


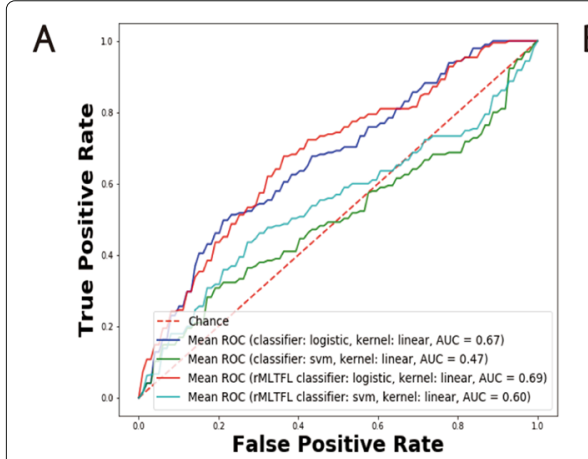

\section{B}
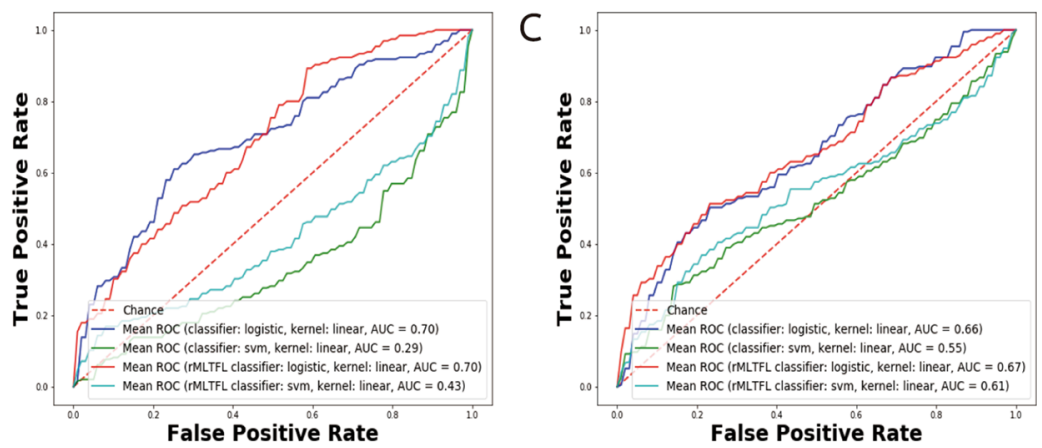

Fig. 7 AUC curves of BAg of logistic regression, SVM, and rMLTFL (logsitic regression and SVM as classifier). Panels $\mathbf{A}$, B, and C correspond to results on the FDG, VBM, and combination of two data modalities respectively


Fig. 8 AUC curves of BAg of logistic regression, SVM, and rMLTFL (logsitic regression and SVM as classifier). Panels $\mathbf{A}$ and $\mathbf{B}$, correspond to results on the validation and testing datasets respectively 


\section{Abbreviations}

AD: Alzheimer's disease; MCl: Mild cognitive impairment; NC: Normal control; L-MCI: Late stage mild cognitive impairment; E-MCl; Early stage mild cognitive impairment; CT: Computed tomography; MRI: Magnetic resonance imaging; PET: Positron emission tomography; VBM: Voxel-based measure; FDG: fluorodeoxyglucosee TL: Transfer learning; ADNI: The Alzheimer's Disease Neuroimaging Initiative; MMSE: Mini-Mental State Examination; CDR: Clinical dementia rating; BAg: Bootstrap aggregation; OT: Optimal transport; rMLTFL: Multi-label transfer feature learning; SVM: Support vector machine; LR: Logistic regression; ANOVA: Analysis of variance; t-SNE: t-distributed stochastic neighbor embedding; SD: Sinkhorn distance; AUC: Area under the receiver operating curve; IW: Importance-weighting; TCA: Transfer component analysis; SUBA: Semi-supervised subspace alignment; FLDA: Feature-level domain adaptation; TrAdaBoost: Boosting for transfer learning; MKL: Multiple kernel learning; AverageMKL: sSmple average of base kernels; EasyMKL: Radius-based combination of kernels; GRAM: Radius-margin ratio optimization for dotproduct boolean kernel learning; RMKL: Radius-based multiple kernel learning; PWMK: Simple but effective methods for combining kernels in computational biology; CKA: Centered kernel alignment optimization in closed form

\section{Supplementary Information}

The online version contains supplementary material available at https://doi.org/10.1186/s13195-021-00915-3.

Additional file 1: This csv file contains the pre-processed FDG features from [18]. Label 1, 3, 4, 5 correspond to NC, E-MCI, L-MCI and AD subjects respectively.

Additional file 2: This csv file contains the pre-processed VBM features from [18]. Label 1, 3, 4, 5 correspond to NC, E-MCI, L-MCl and AD subjects respectively.

Additional file 3: This pdf file contains two tables which include results on two related tasks (table 1, 2).

\section{Acknowledgements}

Not applicable.

\section{Authors' contributions}

Study design: Travis S. Johnson, Ziyu Liu, Shao We, Jie Zhang and Kun Huang; data cleaning and pre-processing: Wei Shao; modeling and computational methods: Ziyu Liu and Wei Shao; paper writing: Travis S. Johnson, Ziyu Liu, and Kun Huang; paper review and supervision: Min Zhang, Jie Zhang, and Kun Huang. The authors read and approved the final manuscript.

\section{Funding}

This work is partially supported by Indiana University Precision Health Initiative (to LZ, TJ) and NIH U54AG065181 grant (to KH, ZJ, WS).

\section{Availability of data and materials}

The dataset(s) supporting the conclusions of this article is(are) included within the article (and its additional file(s).

\section{Declarations}

Ethics approval and consent to participate

Not applicable.

\section{Consent for publication}

Not applicable.

\section{Competing interests}

The authors declare that they have no competing interests.

\footnotetext{
Author details

${ }^{1}$ Department of Statistics, Purdue University, West Lafayette, USA.

${ }^{2}$ Department of Biostatistics and Health Data Science, Indiana University

School of Medicine, Indianapolis, USA. ${ }^{3}$ Department of Medical and Molecular Genetics, Indiana University School of Medicine, Indianapolis, USA.

${ }^{4}$ Regenstrief Institute, Indianapolis, USA.
}

Received: 15 February 2021 Accepted: 5 October 2021

Published online: 07 January 2022

\section{References}

1. Alzheimer's Disease Fact Sheet. U.S. Department of Health and Human Services. https://www.nia.nih.gov/health/alzheimers-disease-fact-sheet. Accessed 10 Feb 2021.

2. Niikura T, Tajima H, Kita Y. Neuronal cell death in alzheimer's disease and a neuroprotective factor, humanin. Curr Neuropharmacol. 2006;4(2): 139-47.

3. Kinney JW, Bemiller SM, Murtishaw AS, Leisgang AM, Salazar AM, Lamb BT. Inflammation as a central mechanism in alzheimer's disease. Alzheimers Dement Transl Res Clin Interv. 2018;4:575-90.

4. Murphy MP, LeVine III H. Alzheimer's disease and the amyloid- $\beta$ peptide. J Alzheimers Dis. 2010;19(1):311-23.

5. Park J-C, Han S-H, Yi D, Byun MS, Lee JH, Jang S, Ko K, Jeon SY, Lee Y-S, Kim YK, et al. Plasma tau/amyloid- $\beta 1-42$ ratio predicts brain tau deposition and neurodegeneration in alzheimer's disease. Brain. 2019;142(3):771-86.

6. Mattson MP. Pathways towards and away from alzheimer's disease. Nature. 2004;430(7000):631-9.

7. How Is Alzheimer's Disease Diagnosed?. U.S. Department of Health and Human Services. https://www.nia.nih.gov/health/how-alzheimersdisease-diagnosed.

8. Edmonds EC, McDonald CR, Marshall A, Thomas KR, Eppig J, Weigand AJ, Delano-Wood L, Galasko DR, Salmon DP, Bondi MW, et al. Early versus late mci: Improved mci staging using a neuropsychological approach. Alzheimers Dement. 2019;15(5):699-708.

9. Jack Jr CR, Bennett DA, Blennow K, Carrillo MC, Dunn B, Haeberlein SB, Holtzman DM, Jagust W, Jessen F, Karlawish J, et al. Nia-aa research framework: toward a biological definition of alzheimer's disease. Alzheimers Dement. 2018;14(4):535-62.

10. Ahmed OB, Benois-Pineau J, Allard M, Catheline G, Amar CB, Initiative $A D N$, et al. Recognition of alzheimer's disease and mild cognitive impairment with multimodal image-derived biomarkers and multiple kernel learning. Neurocomputing. 2017;220:98-110.

11. Cohen AD, Klunk WE. Early detection of alzheimer's disease using pib and fdg pet. Neurobiol Dis. 2014;72:117-22.

12. Zhang $Y$, Dong Z, Phillips $P$, Wang S, Ji G, Yang J, Yuan T-F. Detection of subjects and brain regions related to alzheimer's disease using $3 \mathrm{~d}$ mri scans based on eigenbrain and machine learning. Front Comput Neurosci. 2015;9:66.

13. Cheng B, Liu M, Zhang D, Shen D. Robust multi-label transfer feature learning for early diagnosis of alzheimer's disease. Brain Imaging Behav. 2019;13(1):138-53.

14. Cheng B, Liu M, Suk H-I, Shen D, Zhang D. Multimodal manifold-regularized transfer learning for mci conversion prediction. Brain Imaging Behav. 2015;9(4):913-26.

15. Zhuang F, Qi Z, Duan K, Xi D, Zhu Y, Zhu H, Xiong H, He Q. A comprehensive survey on transfer learning. Proc IEEE. 2020;109(1):43-76.

16. Flamary R, Courty N, Rakotomamonjy A, Tuia D. Optimal transport with laplacian regularization. In: NIPS 2014, Workshop on Optimal Transport and Machine Learning. Cambridge: MIT press; 2014.

17. Courty N, Flamary R, Tuia D, Rakotomamonjy A. Optimal transport for domain adaptation. IEEE Trans Patt Anal Mach Intell. 2016;39(9):1853-65.

18. Hao X, Bao Y, Guo Y, Yu M, Zhang D, Risacher SL, Saykin AJ, Yao X, Shen $\mathrm{L}$, Initiative $A D N$, et al. Multi-modal neuroimaging feature selection with consistent metric constraint for diagnosis of alzheimer's disease. Med Image Anal. 2020;60:101625.

19. Levina $E$, Bickel $P$. The earth mover's distance is the mallows distance: Some insights from statistics. In: Proceedings Eighth IEEE International Conference on Computer Vision. ICCV 2001, vol 2. New York: IEEE; 2001. p. 251-56.

20. Perrot M, Courty N, Flamary R, Habrard A. Mapping estimation for discrete optimal transport. In: Proceedings of the 30th International Conference on Neural Information Processing Systems. Cambridge: MIT press; 2016. p. 4204-12.

21. Peleg $S$, Werman M, Rom H. A unified approach to the change of resolution: Space and gray-level. IEEE Trans Patt Anal Mach Intell. 1989;11(7):739-42. 
22. Breiman L. Bagging predictors. Mach Learn. 1996;24(2):123-40.

23. Bickel $S$, Brückner $M$, Scheffer T. Discriminative learning under covariate shift. J Mach Learn Res. 2009;10(9):2137-55.

24. Pan SJ, Tsang IW, Kwok JT, Yang Q. Domain adaptation via transfer component analysis. IEEE Trans Neural Netw. 2010;22(2):199-210.

25. Yao T, Pan Y, Ngo C-W, Li H, Mei T. Semi-supervised domain adaptation with subspace learning for visual recognition. In: Proceedings of the IEEE Conference on Computer Vision and Pattern Recognition. New York: IEEE; 2015. p. 2142-50.

26. Kouw WM, Van Der Maaten $\amalg$, Krijthe JH, Loog M. Feature-level domain adaptation. J Mach Learn Res. 2016;17(1):5943-74.

27. Yao Y, Doretto G. Boosting for transfer learning with multiple sources. In: 2010 IEEE Computer Society Conference on Computer Vision and Pattern Recognition. New York: IEEE; 2010. p. 1855-62.

28. Aiolli F, Donini M. Easymkl: a scalable multiple kernel learning algorithm. Neurocomputing. 2015;169:215-24.

29. Lauriola I, Polato M, Aiolli F. Radius-margin ratio optimization for dot-product boolean kernel learning. In: International Conference on Artificial Neural Networks. Salmon Tower Building New York: Springer; 2017. p. 183-91.

30. Do H, Kalousis A, Woznica A, Hilario M. Margin and radius based multiple kernel learning. In: Joint European Conference on Machine Learning and Knowledge Discovery in Databases. Salmon Tower Building New York: Springer; 2009. p. 330-43.

31. Tanabe H, Ho TB, Nguyen $\mathrm{CH}$, Kawasaki S. Simple but effective methods for combining kernels in computational biology. In: 2008 IEEE International Conference on Research, Innovation and Vision for the Future in Computing and Communication Technologies. New York: IEEE; 2008. p. 71-8

32. Cortes C, Mohri M, Rostamizadeh A. Two-stage learning kernel algorithms. In: 2010 International Conference on Machine Learning. Haifa; 2010. p. 239-46.

33. Sabbagh MN, Boada M, Borson S, Chilukuri M, Doraiswamy P, Dubois B, Ingram J, Iwata A, Porsteinsson A, Possin K, et al. Rationale for early diagnosis of mild cognitive impairment (mci) supported by emerging digital technologies. J Prev Alzheimers Dis. 2020;7:158-64.

34. Rasmussen J, Langerman H. Alzheimer's disease-why we need early diagnosis. Degenerative Neurol Neuromuscul Dis. 2019;9:123.

35. Wong W. Economic burden of alzheimer disease and managed care considerations. Am J Manag Care. 2020;26(8 Suppl):177-83.

\section{Publisher's Note}

Springer Nature remains neutral with regard to jurisdictional claims in published maps and institutional affiliations.

\section{Ready to submit your research? Choose BMC and benefit from:}

- fast, convenient online submission

- thorough peer review by experienced researchers in your field

- rapid publication on acceptance

- support for research data, including large and complex data types

- gold Open Access which fosters wider collaboration and increased citations

- maximum visibility for your research: over $100 \mathrm{M}$ website views per yea

At BMC, research is always in progress.

Learn more biomedcentral.com/submission 